\title{
Una revisión de los modelos de volatilidad estocástica
}

\author{
A revision of stochastic volatility models
}

\author{
Ronne Tamayo Medina ${ }^{\mathrm{a}}$ \\ Heivar Yesid Rodriguez Pinzón ${ }^{b}$ \\ ronne0004@yahoo.fr \\ heivarrodriguez@usantotomas.edu.co
}

\begin{abstract}
Resumen
En economía, una buena parte de los procesos observados a través del tiempo se plantean como el resultado de efectos de variables latentes, es decir, procesos no observables de forma directa. Este es el caso de la volatilidad de la rentabilidad en el mercado financiero, la cual ha sido modelada desde comienzos de los años $80 \mathrm{em}$ pleando modelos de varianza condicional ARCH y GARCH y, más recientemente modelos de volatilidad estocástica SV, los cuales presentan un menor número de parámetros que los modelos GARCH y permiten estudiar la naturaleza no-lineal de la volatilidad.Debido a que en el modelo SV no se conoce de forma exácta la función de verosimilitud, se emplea el método de estimación máximo cuasi-verosímil propuesto por Geert \& Campbell (1994), el cual utiliza la representación en forma de modelo de estados State-Space. La representacion del modelo SV mediante la forma de estados se evalua a traves de filtros adaptativos, como es el caso de los filtros Kalman, lo cual implica un mayor costo computacional. A partir de lo anterior, no necesariamente se llega a la solución óptima del problema.
\end{abstract}

Palabras clave: Filtro Kalman, modelos de estado-espacio, modelos de volatilidad estocástica.

\begin{abstract}
In economics, a good part of the processes observed over time arise as the result of effects of latent variables, ie processes not directly observable. This is the case of the volatility of financial market returns, which has been shaped since the early 80 s using $\mathrm{ARCH}$ and GARCH conditional variance models, and more recently stochastic volatility models (SV), which present fewer parameters than GARCH models and allow us to study the non-linear nature of volatility. Because in the SV model is not known accurately the likelihood function, the method of maximum
\end{abstract}

\footnotetext{
a Estadístico. Universidad Nacional de Colombia.

${ }^{\mathrm{b}}$ Profesor. Facultad de Estadística. Universidad Santo Tomás.
} 
quasi-likelihood is used. This method uses the representation in state-space model form. The SV model representation is evaluated through adaptive filters, such as Kalman, which implies a higher computational cost.

Key words: Kalman filter, state-space models, volatility models.

\section{Introducción}

El paradigma central de las finanzas consiste en asumir ciertos riesgos para obtener beneficios, pero no todos los riesgos aportan las mismas compensaciones. Tanto los riesgos como los beneficios pertenecen al futuro. Es así como se busca minimizar los riesgos de las pérdidas previstas al tiempo que se desea maximizar los rendimientos esperados. Ideas que adquirieron un nuevo significado desde que Markowitz (1952), Tobin (1958) y posteriormente Sharpe (1964), asociaron el riesgo a la varianza del valor de una cartera, basándose en la búsqueda de la evasión del riesgo, teoría a la cual denominaron valoración de los precios de los activos de capital (CAPM). Este modelo muestra que existe una relación natural entre los rendimientos esperados y la varianza. Luego, Black \& Scholes (1973) y Merton (1974) desarrollaron un modelo para evaluar el precio de las opciones.

Las opciones proporcionan a su propietario el derecho de vender un activo a un precio determinado en un momento del futuro. En este sentido, las opciones pueden ser consideradas como un seguro, pero el precio de este seguro depende de los riesgos y estos riesgos se miden a partir de la varianza de los rendimientos del activo. Por otra parte, la volatilidad de un periodo futuro es la medida de riesgo que debería considerarse, por lo que son necesarias no sólo una medida de la volatilidad actual, sino también una predicción de la volatilidad futura. Teniendo en cuenta estos aspectos, se desarrolló una nueva metodología en los estudios de la volatilidad, a partir del comportamiento en la rentabilidad de los activos. Razón por la cual se presenta el caso discreto como el continuo para la rentabilidad.

\subsection{La rentabilidad}

Las variaciones en el precio de apertura y cierre de un índice son la causa de la rentabilidad. Los rendimientos son prácticamente impredecibles, tienen una gran cantidad de valores extremos y los periodos de mayor agitación como los de tranquilidad están agrupados en el tiempo. Estas características a menudo se describen con exceso de curtosis y agrupamiento de la volatilidad. Es decir, cuando la volatilidad es elevada, es probable que permanezca en alza e igual situación cuando esta tiende a la baja. Sin embargo, estos periodos están limitados en el tiempo, así que es seguro que la predicción terminara acercándose hacia volatilidades me-

nos extremas. Las dos formas más usuales en el cálculo de la rentabilidad son la rentabi-lidad compuesta y la rentabilidad continua, conceptos que se presentan, para una mejor compresión del trabajo. 


\subsection{Rentabilidad compuesta}

Sean $P_{t-1}$ y $P_{t}$ el precio de cierre del índice en los periodos $t-1$ y $t$ respectivamente. La rentabilidad $R_{t}$ obtenida en el periodo $t$ está dada por:

$$
R_{t}=\frac{P_{t}-P_{t-1}}{P_{t-1}}=\frac{P_{t}}{P_{t-1}}-1, t=1,2, \ldots T
$$

La rentabilidad en $k$-periodos, está dada por:

$$
R_{t}(k)=\frac{P_{t}}{P_{t-k}}-1, t=1,2, \ldots T
$$

\subsection{Rentabilidad continua}

Sean $P_{t-1}$ y $P_{t}$ el precio de cierre del índice en los periodos $t-1$ y $t$ respectivamente. La rentabilidad $R_{t}$ continua del periodo $t$ se define como:

$$
r_{t}=\operatorname{Ln}\left(\frac{P_{t}}{P_{t-1}}\right), t=1,2, \ldots T
$$

La rentabilidad en $k$-periodos, está dada por:

$$
r_{t}(k)=\operatorname{Ln}\left(\frac{P_{t}}{P_{t-k}}\right)=r_{t}+r_{t-1}+\ldots+r_{t-k+1}, t=1,2, \ldots T
$$

Ambos tipos de rentabilidad, se relacionan mediante la expresión:

$$
r_{t}(k)=\operatorname{Ln}\left\{\prod\left(1+R_{t-i}\right)\right\}, t=1,2, \ldots T
$$

Esta última expresión, permite analizar el comportamiento discreto de la variable rentabilidad desde una perspectiva continua, con el fin de que sea modelada a través de un proceso autoregresivo.

\section{Volatilidad estocástica}

La volatilidad asociada a una serie de rentabilidades no es observable; por tanto, el estudiar el comportamiento de la serie de volatilidades requiere de un proceso de estimación. La forma más simple de medir la volatilidad se basa en el cálculo de la desviación típica asociada a un conjunto de rentabilidades observadas. Así, se obtiene un único valor que representa la dispersión global de los datos, pero no la evolución de esta dispersión a lo largo del tiempo. En la literatura financiera, se define la volatilidad como una medida de la intensidad de los cambios aleatorios o impredecibles en la rentabilidad o en el precio futuro de un titulo. En la representación grafica de una serie histórica de rendimientos, se asocia la volatilidad con la amplitud de las fluctuaciones del mismo. En general, la volatilidad se caracteriza: 
- Exceso de curtosis (Mandelbrot 1963) y (Fama 1965)

- Periodos de alta y baja volatilidad, también conocidos como conglomerados de volatilidad (volatility clusters), es decir, si la volatilidad es baja o elevada en un periodo, tiende a seguir este comportamiento (Mandelbrot 1963) y (Engle 1982).

- De manera ocasional, se producen valores altos de volatilidad, que se interpretan como discontinuidades de salto en los precios (Figlewski 1997).

- Periodos de alta o baja volatilidad tienden a estar precedidos de periodos en los que la volatilidad es más moderada a largo plazo (Hsieh 1995) y (Figlewski 1997).

- Comportamiento asimétrico de las series de volatilidades, según informaciones de los mercados financieros (Campbell \& Hentschel 1992).

- Movimientos conjuntos de la volatilidad, es decir, que al estudiar en diferentes mercados series distintas, pero del mismo concepto (cotizaciones, tipos de interés, entre otros), se observa que los movimientos importantes en un mercado están relacionados con movimientos importantes en otro mercado (Aydemir 1998).

Para realizar la estimación de la volatilidad, se cuenta con métodos paramétricos y no-paramétricos, estos últimos poseen la ventaja de que necesitan muy pocas hipótesis para obtener las estimaciones de la volatilidad. Entre los métodos paramétricos, se cuentan los basados en el cálculo de medias móviles y los que asumen un modelo para estimar la volatilidad. Sin embargo, existen otros métodos para calcular la rentabilidad basada en otros precios. Por ejemplo, (Garman \& Klass 1980), estiman la volatilidad de un día a partir de la diferencia entre el precio más alto y el más bajo del día.

La volatilidad diaria $\widehat{\sigma}^{2}$, se puede definir:

$$
\widehat{\sigma}^{2}=\frac{0,361}{n} \sum_{i=1}^{n}\left(H_{i}-L_{i}\right)^{2}
$$

Donde $H_{i}$ es el precio máximo, $L_{i}$ es el precio mínimo y $n$ es el número de días.

\subsection{Modelos GARCH}

Engle (1982), inicialmente presentó un caso particular, los modelos ARCH y posteriormente Bollerslev (1986), presenta la forma general GARCH. En los modelos GARCH, se plantea la varianza como una estimación de la volatilidad, esto a través de dos ecuaciones. La primera ecuación explica la evolución los rendimientos en función de rendimientos pasados y la segunda ecuación modela la rentabilidad. La expresión matemática de este modelo es: 
Sea $\left\{Y_{t}\right\}$ un proceso de rendimientos observados, que obedece la ecuación:

$$
Y_{t}=\beta_{0}+\beta_{1} Y_{t-1}+\beta_{2} Y_{t-2}+\ldots+\beta_{p} Y_{t-p}+\mu_{t}
$$

Donde:

$$
\mu_{t}=\sqrt{h_{t}} v_{t}
$$

y

$$
\mu_{t}=\left\{v_{t}\right\} \sim N I I D(0,1)
$$

con $\left\{\mu_{t}\right\}$, es un proceso GARCH y se denota $\mu_{t} \sim G A R C H(r, m)$, si obedece la ecuación:

$$
h_{t}=a_{0}+a_{1} h_{t-1}+a_{2} h_{t-2}+\ldots+a_{r} h_{t-r}+c_{1} \mu_{t-1}^{2}+c_{2} \mu_{t-2}^{2}+\ldots+c_{m} \mu_{t-r_{k}}^{2}(8)
$$

El modelo más utilizado en el cálculo de la volatilidad es el $G A R C H(1,1)$, para este modelo la ecuación (7), se reduce a:

$$
h_{t}=a_{0}+a_{1} h_{t-1}+c_{1} \mu_{t-1}^{2}
$$

Los modelos de la familia GARCH, presentan problemas relacionados con la predicción Figlewski (1997), debido a que necesitan un gran número de datos para obte-ner una estimación robusta y no están diseñados para generar predicciones de la varianza varios pasos adelante, lo cual genera inconvenientes en las estimaciones, puesto que períodos de alta volatilidad suelen estar precedidos de períodos de baja volatilidad y viceversa, es decir que el efecto de una perturbación aleatoria en la volatilidad tiene efectos duraderos en el tiempo. En este sentido, los modelos con memoria larga DTARCH, han resultado más eficientes para modelar la estructura dinámica de la volatilidad; de estos se presentan a continuación sus características más importantes.

\subsection{Modelos DTARCH}

Para considerar los efectos duraderos de una perturbación aleatoria en la volatilidad, en la modelación de la varianza condicionada Li \& Li (1996), proponen el modelo ARCH de doble umbral DTARCH, el cual combina las características de los modelos TAR, propuestos por (Tong 1978) y de los modelos ARCH. Estos modelos DTARCH, se caracterizan porque la esperanza condicionada posee una estructura lineal dentro de cada régimen y la varianza condicionada cambia a partir de unos niveles previos de la serie.

Se dice que un proceso $\left\{Y_{t}\right\}$ sigue un modelo DTARCH, con parámetros $\left(l_{1}, p_{1}, \ldots, p_{l 1}, l_{2}, q_{1}, \ldots, q_{l 2}\right)$, si satisface la ecuación:

$$
Y_{t}=\sum_{j=1}^{l_{1}}\left[\phi_{0}^{(j)}+\sum_{i=1}^{p_{j}} \phi_{i}^{(j)} Y_{t-1}\right] 1_{\left(Y_{t-d} \in R_{j}\right)}+\varepsilon_{t}
$$


Donde :

$$
\begin{gathered}
E\left(\varepsilon_{t} / F_{t-1}\right)=0, \sigma_{0}^{(k)}>0, F_{t-1}=\sigma\left(Y_{t-1}, \ldots\right) \\
\operatorname{Var}\left(\varepsilon_{t} / F_{t-1}\right)=h_{t}=\left[\sum_{k=1}^{l_{2}} \sigma_{0}^{(k)}+\sum_{r=1}^{q_{k}} \sum_{s=1}^{q_{k}} \sigma_{r s}^{(k)} \varepsilon_{t-r} \varepsilon_{t-s}\right] 1_{\left(\varepsilon_{t-d^{*}} \in R_{k}^{*}\right)}
\end{gathered}
$$

A su vez :

$$
R_{j}=\left(r_{j-1,}, r_{j}, j=1, \ldots l_{1}, R_{k}^{*}=\left(r_{k-1}^{*}, r_{k}^{*}\right\rceil, k=1, \ldots l_{2}\right.
$$

$\left\{R_{j}\right\}$ y $\left\{R_{k}^{*}\right\}$ son dos particiones diferentes de la recta real, $\sum^{(k)}=\left[\sigma_{r s}^{(k)}\right]$ es definida no negativa, $d$ y $d^{*}$ son parámetros de retardo, no negativos. El modelo DTARCH, no es fácil de implementar y posee un gran número de parámetros, lo cual implica disponer de un gran número de observaciones y esto no siempre se tiene. La familia GARCH, considera a la volatilidad como una función determinística de las observaciones pasadas, es decir, la varianza condicionada se mantiene fija y no tiene en cuenta factores aleatorios, lo cual hace a estos modelos ineficientes en la fase de predicción (Rabemananjara \& Zakoian 1993). Una posible alternativa para resolver los inconvenientes anteriores, son los modelos de volatilidad estocástica, que se presentan a continuación y que son el centro de interés de este trabajo.

\subsection{Modelos de volatilidad estocástica}

Una posible solución a los inconvenientes observados en los modelos anteriores, se plantea a través del modelo de volatilidad estocástica SV, introducido por Taylor (1986) y Shephard (1986), en donde se supone que la rentabilidad depende de una variable no observable (variable latente), que sigue un proceso estocástico ARMA y de una variable aleatoria independiente e idénticamente distribuida. En general, se tiene que las rentabilidades observadas $\left\{Y_{t}\right\}$, se expresan:

$$
Y_{t}=\sigma_{t} \epsilon_{t}, \varepsilon_{t} \sim \operatorname{IID}(0,1)
$$

Los modelos SV, especifican el algoritmo de la volatilidad como un proceso estocástico lineal. En la versión más simple, se tiene que la solución para este modelo se construye a partir de su representación de un modelo $A R(1)$. De la ecuación (13), elevando al cuadrado y evaluando $L n$ se obtiene:

$$
\operatorname{Ln} Y_{t}^{2}=\operatorname{Ln} \sigma_{t}^{2}+\operatorname{Ln} \epsilon_{t}^{2}, \varepsilon_{t} \sim \operatorname{IID}(0,1)
$$


y la volatilidad $\sigma_{t}^{2}$ obedece la ecuación:

$$
\operatorname{Ln} \sigma_{t}^{2}=\beta_{0}+\beta_{1} \operatorname{Ln}\left(\sigma_{t-1}^{2}\right)+w_{t},\left\{w_{t}\right\} \sim N I D\left(0, \sigma_{w}\right)
$$

Los modelos ARCH y los modelos SV, difieren en la definición de la ecuación que modela las volatilidades. En los primeros a la volatilidad se le considera una función no-estocástica lineal del comportamiento pasado de la serie, mientras que en los modelos SV, la volatilidad se considera una componente no observable de la serie, modelada a través de un proceso lineal autorregresivo. Dos trabajos recientes acerca de estos modelos es el de (Breidt et al. 1998), autor que considera el modelo de volatilidad estocástica de larga memoria LMSV. Desde el punto de vista financiero, los modelos SV son atractivos dado que constituyen una aproximación en tiempo discreto a los modelos en tiempo continuo utilizados en finanzas. Sin embargo, su estimación resulta más complicada que en los modelos GARCH, ya que no se puede construir de forma exacta la función de verosimilitud, lo cual hace necesario la utilización de métodos de estimación alternativos tales como: estimación de máxima verosimilitud simulada, estimación por inferencia indirecta, el método generalizado de momentos y finalmente la estimación máximo cuasi-verosímil.

El método de estimación máximo cuasi-verosímil, propuesto por Harvey (1994), utiliza la representación en forma de modelo de estados (State-Space), que admiten los modelos SV para llegar a los estimados deseados. Con esta estructura, el modelo puede estimarse, tratando las perturbaciones del modelo, bajo distribución normal y empleando el filtro de Kalman para obtener la descomposición de la verosimilitud en los errores de predicción.

\section{Modelos de estado}

Entre los usos particulares de los modelos de estado (state-space model), se encuentra la modelación de componentes no observables, que pueden incluir variables latentes, tales como el ciclo económico, la tasa natural de crecimiento de la población, la volatilidad en retornos de precios o expectativas inflacionarias. Aunque las componentes del proceso no sean directamente observables, su comportamiento es de gran interés en campos como la econometría y el control de sistemas lineales como se evidencia en los trabajos de (Davis \& Vinter 1985) y (Hannan \& Deistler 1988). También permiten ajustar modelos con parámetros que cambian en el tiempo, los cuales son muy útiles cuando se analizan cambios estructurales, como en el estudio de (Durbin \& Harvey 1985), sobre el efecto del uso de cinturones de seguridad en accidentes de tránsito en la Gran Bretaña en donde la representación de una serie de observaciones a través del tiempo, se describe en términos de sus componentes.

$$
Y_{t}=\mu_{t}+\gamma_{t}+\epsilon_{t}
$$


donde:

- $\mu_{t}$ : Componente de tendencia

- $\gamma_{t}$ : Componente de estacionalidad

- $\epsilon_{t}$ : Componente de Ruido Aleatorio

Un modelo de estado está caracterizado por dos ecuaciones. La primera es conocida como ecuación de observación. Corresponderá a la ecuación (17), la cual expresa al vector de observaciones como una función lineal de variables de estado mas un ruido aleatorio.

En un modelo estructural de series temporales, los elementos del vector de estado $X_{t}$ son los componentes no observables de la serie.

$$
Y_{t}=F_{t} X_{t}+\mu_{t}, t=1,2 \ldots
$$

y $\left\{\mu_{t}\right\} \sim R . B$. La segunda ecuación, se denomina ecuación de estado y corresponderá a la ecuación (18), la cual determina el estado de la variable en el tiempo $t$ en términos del estado anterior mas un término de ruido, obteniendo.

$$
X_{t}=G_{t} X_{t-1}+w_{t}, t=1,2 \ldots
$$

y $\left\{w_{t}\right\} \sim R . B$. A su vez, se asume que el estado inicial es una variable no correlacionada con los términos de ruido $y$, asimismo, los ruidos son no correlacionados entre sí, es decir:

$$
\begin{aligned}
& \operatorname{Cov}\left(\mu_{t}, w_{t}\right)=0 \\
& \operatorname{Cov}\left(\mu_{t}, x_{t}\right)=0 \\
& \operatorname{Cov}\left(w_{t}, x_{0}\right)=0
\end{aligned}
$$

El modelo SV, que consiste en la ecuacion (15), se transforma a su representación en modelo de estados, a través de las ecuaciones (17) y (18) respectivamente. El propósito del análisis en el espacio de los estados es inferir acerca de las propiedades relevantes de los estados, en este caso $X_{1}, \ldots, X_{t}$ a partir de los valores observados $Y_{1}, \ldots Y_{t}$. Para ello, es preciso establecer una relación entre los estados en los momentos $t$ y $t-1$, es decir,

$$
\operatorname{Ln} \sigma_{t}^{2}=\beta_{0}+\beta_{1} \operatorname{Ln}\left(\sigma_{t-1}^{2}\right)+w_{t},\left\{w_{t}\right\} \sim N I D\left(0, \sigma_{w}\right)
$$

De la ecuación (13) se obtiene:

$$
\operatorname{Ln} Y_{t}^{2}=\operatorname{Ln} \sigma_{t}^{2}+\operatorname{Ln} \epsilon_{t}^{2}, \varepsilon_{t} \sim \operatorname{IID}(0,1)
$$

Ahora, se redefinen las nuevas variables $y_{t}, x_{t}$ y $n_{t}$ a partir de la ecuación (14), obteniendo las siguientes ecuaciones:

$$
\begin{aligned}
y_{t} & =\operatorname{Ln} Y_{t}^{2} \\
x_{t} & =\operatorname{Ln} \sigma_{t}^{2} \\
y_{t} & =x_{t}+n_{t}, n_{t} \sim \operatorname{Ln}_{t}^{2}
\end{aligned}
$$


Ecuación con la cual se estima el valor del estado, es decir, la magnitud que evoluciona en el tiempo y que no se observa directamente, sino contaminada por el factor de ruido. De la ecuación (26), se tiene:

$$
y_{t}=x_{t}+n_{t}
$$

con $n_{t} \approx L n \epsilon_{t}^{2} \sim\left(-1.27, \frac{\pi^{2}}{2}\right)$. Donde, $n_{t}$ no sigue una distribución normal, pero en donde se conoce su media y varianza, Abramovitz (1970).

Ahora, la ecuación (15) se redefine teniendo en cuenta la transformación (25), obteniendo:

$$
x_{t}=\beta_{0}+\beta_{1} x_{t-1}+w_{t}, w_{t} \sim N I D(0,1)
$$

Donde $\beta_{0}$ es alguna transformación lineal. A partir de lo anterior, se construyen las expresiones matriciales de transición, para el modelo de estados. Luego, para la ecuacion (28), se tiene:

$$
x_{t}=\left(\begin{array}{cc}
\beta_{0} & \beta_{1} \\
0 & 1
\end{array}\right)\left(\begin{array}{c}
1 \\
x_{t-1}
\end{array}\right)+\left(\begin{array}{c}
0 \\
w_{t}
\end{array}\right)
$$

la cual, a partir de la ecuación (18), se puede reescribir en la forma:

$$
x_{t}=G x_{t-1}+\xi_{t}, \xi_{t}=\left(\begin{array}{c}
0 \\
w_{t}
\end{array}\right)
$$

A su vez, para la transformación empleada en la ecuacion (24), se obtiene la representación en el modelo de estados, a través de la expresión:

$$
y_{t}=\left(\begin{array}{ll}
0 & 1
\end{array}\right)\left(\begin{array}{c}
1 \\
x_{t}
\end{array}\right)+n_{t}
$$

Lo cual se puede denotar en la forma de estados de la ecuación (17):

$$
y_{t}=F x_{t}+n_{t}
$$

Llegando así, las ecuaciones de volatilidad a la forma del sistema de modelo de estados:

$$
\begin{aligned}
& y_{t}=F x_{t}+n_{t} \\
& x_{t}=G x_{t-1}+\xi_{t}
\end{aligned}
$$

Donde $F$, al igual que $G$, son transformaciones lineales que describen la evolución en el tiempo del sistema. Las matrices $F$ y $G$ se denominan matrices de sistema y sus valores se asumen conocidos. Sin embargo, las anteriores matrices se consideran no estocásticas, aunque podrían cambiar a lo largo del tiempo de forma 
predeterminada. De esta manera, el sistema es lineal y para cualquier momento del tiempo, el vector de variables observables $y_{t}$, se puede expresar como una combinación lineal de valores presentes y pasados de perturbaciones y del vector de estado inicial $\beta_{0}$.

Si las matrices del sistema no cambian con el tiempo, el modelo se denomina homogéneo o invariante respecto al tiempo y los modelos no estacionarios constituyen casos particulares que, siendo invariantes respecto al tiempo, se transforman en estacionarios al aplicarles el operador diferencia. Las matrices del sistema, pueden depender de un conjunto de parámetros desconocidos que determinan las propiedades estocásticas del modelo y se denominan hiperparámetros.

Nótese que la estacionariedad en media del estado se produce si $\beta_{0}$, o bien, si $G$ posee algún valor propio unitario tal que $G \beta_{0}$ es constante. Por otra parte, se puede demostrar que los momentos de segundo orden son invariantes respecto a $t$ sí todos los autovalores de $G$ se encuentran dentro del círculo unitario Steyn (1996).

\section{Filtro Kalman}

El origen del filtro Kalman y sus derivaciones, se remonta al concepto de regresión de mínimos cuadrados que se asocia por una parte con Legendre (1752-1833), quien publicó la teoría en 1805 y acuñó el término de mínimos cuadrados. Sin embargo, fue Gauss (1777-1855) quien desarrolló el método como un instrumento estadístico incorporando los mínimos cuadrados en un contexto en el que se da un tratamiento probabilístico a los errores. La relevancia de la exposición de Gauss para la teoría de estimación de mínimos cuadrados recursivos y para el concepto del filtro de Kalman se encuentra en un breve párrafo donde Gauss muestra que es posible detectar los cambios más probables de un evento desconocido cuando una nueva ecuación es incorporada. Razon por la cual se le denomina filtro adaptativo, debido a sus características de autoajuste, es decir, permite corregir estimaciones cuando se recibe nueva información.

Se define al filtro de Kalman como un conjunto de ecuaciones matemáticas que proveen una solución recursiva eficiente del método de mínimos cuadrados. El término recursivo significa que el filtro recalcula la solución cada vez que una nueva observación o medida es incorporada en el sistema. Esta solución permite calcular un estimador lineal, insesgado y óptimo del estado de un proceso en cada momento del tiempo con base en la información disponible en el momento $t-1$, y actualizar, con la información adicional disponible en el momento t, dichas estimaciones. Este filtro es el principal algoritmo para estimar sistemas dinámicos especificados en la forma de estado-espacio.

El algoritmo conocido como filtro Kalman permite obtener información sobre el estado $X_{t}$, a partir de la observación de la variable $Y_{t}$, relacionadas mediante dos enfoques diferentes. El primero, tiene por objeto encontrar un estimador insesgado del estado, que minimice el error cuadrático medio (Harvey 1994) y el segundo, 
se formula en términos más amplios, ya que pretende obtener la distribución del estado $X_{t}$ condicionado a la información observada $I_{t}=\left\{Y_{1}, \ldots, Y_{t}\right\}$, dado que el estimador insesgado de error cuadrático medio mínimo, viene dado por la media de la distribución condicionada, obtenida bajo el supuesto de normalidad Steyn (1996). En ambos enfoques, las aproximaciones son esencialmente iguales.

A partir del conjunto de información $I_{t}$, empleado en la obtención de la distribución condicionada del estado $X_{t}$, a continuación se definen tres casos: en el primero de ellos la solución al problema de predicción ofrecerá estimaciones que son extrapolaciones hacia el futuro de los componentes del vector de estado; por otra parte, el filtrado permitirá actualizar las estimaciones del estado no observable a medida que se dispone de una nueva observación; por último, el suavisado ofrece la mejor estimación del estado en cualquier instante del tiempo con toda la información de la muestra.

\subsection{Predicción para $k<t$}

Para el modelo de estados descrito mediante las ecuaciones (17) y (18), la predicción un paso adelante para el vector de estados $\widehat{X}_{t}=P_{t-1}\left(X_{t}\right)$ y su matriz de covarianzas para el error, se denota.

$$
\begin{aligned}
& \Omega_{t}=E\left[\left(X_{t}-\widehat{X}_{t}\right)\left(X_{t}-\widehat{X}_{t}\right)^{t}\right] \\
& X_{1}=P\left(X_{1} / Y_{0}\right), \Omega_{1}=E\left[\left(X_{1}-\widehat{X}_{1}\right)\left(X_{1}-\widehat{X}_{1}\right)^{t}\right]
\end{aligned}
$$

Donde, las iteraciones para $t=1, \ldots$ se definen de la siguiente forma:

$$
\begin{aligned}
\widehat{X}_{t+1} & =F_{t} \widehat{X}_{t}+\Theta_{t} \Delta_{t}^{-1}\left(Y_{t}-G_{t} \widehat{X}_{t}\right) \\
\Omega_{t+1} & =F_{t} \Omega_{t} F_{t}^{\prime}+Q_{t}-\Theta_{t} \Delta_{t}^{-1} \Theta_{t}^{\prime}
\end{aligned}
$$

Donde:

$$
\begin{aligned}
\Delta_{t} & =G_{t} \Omega_{t} G_{t}^{\prime}+R_{t} \\
\Theta_{t} & =F_{t} \Omega_{t} G_{t}
\end{aligned}
$$

y $\Delta_{t}^{-1}$ es cualquier inversa generalizada de $\Delta_{t}$.

\subsection{Filtrado para $k=t$}

En las estimaciones para el filtro, se tiene: $X_{t / t}=P_{t}\left(X_{t}\right)$ y la matriz de covarianzas para el error, se denota:

$$
\Omega_{t / t}=E\left[\left(X_{t}-X_{t / t}\right)\left(X_{t}-X_{t / t}\right)^{t}\right]
$$

y son determinadas por las relaciones

$$
\begin{aligned}
P_{t} X_{t} & =P_{t-1} X_{t}+\Omega_{t} G_{t}^{\prime} \Delta_{t}^{-1}\left(Y_{t}-G_{t} \widehat{X_{t}}\right) \\
\Omega_{t / t} & =\Omega_{t}-\Omega_{t} G_{t}^{\prime} \Delta_{t}^{-1} G_{t} \Omega_{t}^{\prime}
\end{aligned}
$$




\subsection{Suavizamiento para $k>t$}

Las estimaciones para el ajuste $X_{t / n}=P_{n} X_{t}$ y la matriz de covarianzas para el error

$$
\Omega_{t / n}=E\left[\left(X_{t}-X_{t / n}\right)\left(X_{t}-X_{t / n}\right)^{t}\right]
$$

Son determinadas por el cambio de $t$ por las siguientes iteraciones, las cuales pueden ser resueltas sucesivamente, mediante $n=t, t+1 \ldots$

$$
\begin{aligned}
P_{n} X_{t} & =P_{n-1} X_{t}+\Omega_{t . n} G_{n}^{\prime} \Delta_{n}^{-1}\left(Y_{n}-G_{n} \widehat{X}_{n}\right) \\
\Omega_{t . n+1} & =\Omega_{t . n}\left[F_{n}-\Theta_{n} \Delta_{n}^{-1} G_{n}\right]^{t} \\
\Omega_{t / n} & =\Omega_{t / n-1}-\Omega_{t . n} G_{n}^{\prime} \Delta_{n}^{-1} G_{n} \Omega_{t . n}^{\prime}
\end{aligned}
$$

Con las condiciones iniciales,

$$
\begin{aligned}
P_{t-1} X_{t} & =\widehat{X}_{t} \\
\Omega_{t . t} & =\Omega_{t / t-1}=\Omega_{t}
\end{aligned}
$$

\section{Aplicación al caso colombiano}

Con la fusión de las bolsas de valores de Bogotá, Medellín y Cali en la nueva Bolsa de Valores de Colombia, se centralizó la información acerca de las operaciones en acciones, reduciendo costos de transacción y unificando la cotización de las acciones en el país, lo cual hizo necesario reemplazar los índices de acciones de las tres bolsas existentes, dando origen a un nuevo índice denominado Índice General Bolsa Colombia (IGBC). El IGBC está concebido, al igual que todos los índices de acciones, como un indicador de la evolución de los precios de las acciones más representativas del mercado. Para el cálculo del índice se selecciona una serie de acciones bajo dos criterios:

- La rotación de la acción en el último semestre sea mayor o igual al 0.5.

- La frecuencia de negociación en el trimestre inmediatamente anterior sea superior o igual al $40 \%$.

Los precios del IGBC serán ponderados por un nuevo factor que enlace el índice anterior con el nuevo para garantizar que este sólo se verá afectado por el cambio de precios, es decir, sin incluir variaciones por el cambio de ponderadores y por factores exógenos del mercado. La utilización de este factor permite filtrar el índice de ruidos que impiden reflejar directamente el cambio de precios, lo cual debería en principio quitarle volatilidad al mismo respecto a los índices existentes. Sin embargo, los precios de las acciones colombianas son bastante cambiantes y no 
precisamente por el movimiento de sus fundamentales sino por la carencia de inversiones de largo plazo, ya que muchas veces son especulativas.

El índice se desarrolló como un modelo matemático que busca que el precio de las acciones se pueda predecir con el valor del índice en un momento del tiempo. Se define como el promedio ponderado de los precios para las acciones más representativas del mercado.

$$
\pi_{i}(t)=B_{i} I(t)
$$

Donde:

- $B_{i}$ : Es el precio de la acción estimado en el tiempo t.

- $\pi_{i}$ : Es una constante de proporcionalidad.

- $I(t): \sum W_{j} P_{j}(t)$, donde $W_{i}$ es el ponderador de precios.

La solución del modelo se realiza con la minimización de la Suma Ponderada de los Cuadrados de los Errores-SPCE para la canasta de acciones en todos los instantes del tiempo.

$$
S P C E=\sum \sum \alpha_{I}\left(\pi_{i}(t)-P_{i}(t)\right)^{2}
$$

donde $\alpha_{i}$ es el valor de la ponderacion de los errores según su importancia. Entonces, el índice se define como la sumatoria del precio de cada acción que conforma la canasta por el peso que tiene dentro de la canasta, que resulta de multiplicar $W_{i}^{k}$ por $E$.

$$
I^{k}(t)=E \sum W_{i}^{k} P_{i}(t)
$$

Donde:

- $I^{k}(t)$ : Valor del índice para $t$.

- $t$ : Día o instante en el cual se calcula el índice.

- $k$ : Identifica el trimestre en el que $W_{i}^{k}$ está vigente.

- E : Multiplicatoria de los factores de enlace.

- $W_{i}^{k}$ : Peso o ponderación para la acción $i$, fijo durante $k$.

- $P_{i}(t)$ : Precio de cierre vigente de la acción $i$ en $t$. 


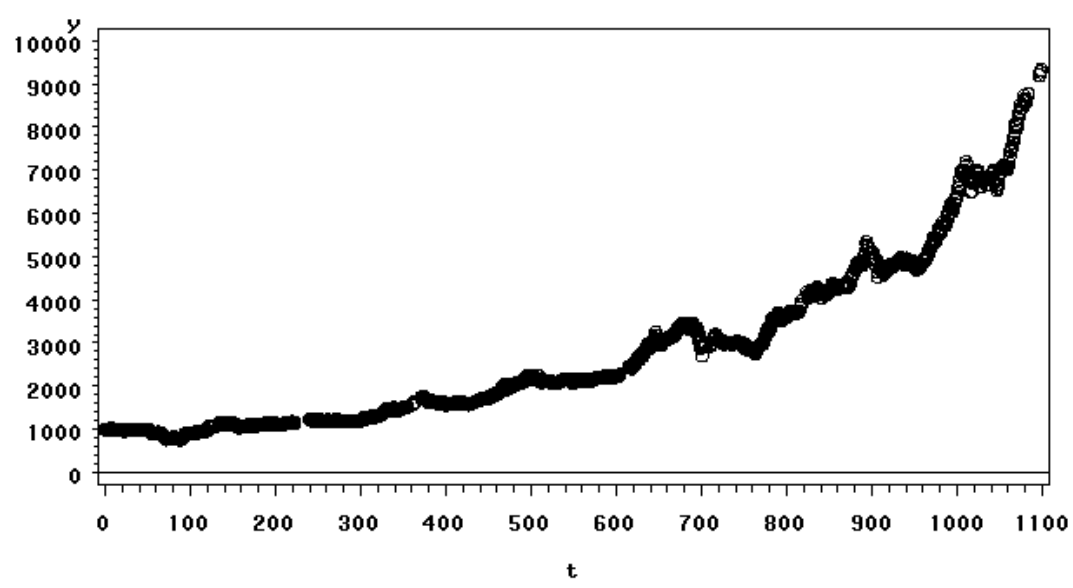

Figura 1: Serie $I G B C$.

Las observaciones registradas a partir del martes 3 de julio de 2001 hasta el viernes 30 de diciembre de 2005 (1100 en total), se representan mediante el siguiente gráfico.

Es de resaltar que los valores corresponden al comportamiento, para la variable IGBC, entre los días lunes a viernes y que el factor "fin de semana" no será considerado. Considerando que los valores de la variable, pertenecen a la categoria de variable continua, se emplea la aproximación para tiempo discreto de la variable (IGBC).

Retomando la ecuación (3)

$$
r_{t}=\operatorname{Ln}\left(\frac{P_{t}}{P_{t-1}}\right), t=1,2, \ldots T
$$

Esta transformación permite eliminar el problema de nivel de la serie, es decir, la "tendencia" positiva y que se representa mediante el siguiente gráfico.

La transformación a su vez, permite analizar el proceso que en principio era de naturaleza no-estacionaria, ahora como un proceso estacionario, al cual es posible ajustar un modelo autoregresivo $\mathrm{AR}(1)$. Es de resaltar que entre las ventajas del Filtro Kalman, se encuentra al aproximación en tiempo continuo a variables registrades en forma discreta (dias).

Para el ajuste del modelo, se propone un modelo AR(1), y con los parámetros de este, se obtienen los valores del filtro. Luego a este filtro se le ajusta un modelo $\mathrm{AR}(1)$ y con estos valores del filtro, se consideran los valores del estado inicial (matriz de estados) y así sucesivamente se realiza el proceso de estimación de los parámetros que hacen parte de la matriz de transición, hasta obtener una diferencia mínima entre los cambios de los parámetros. 


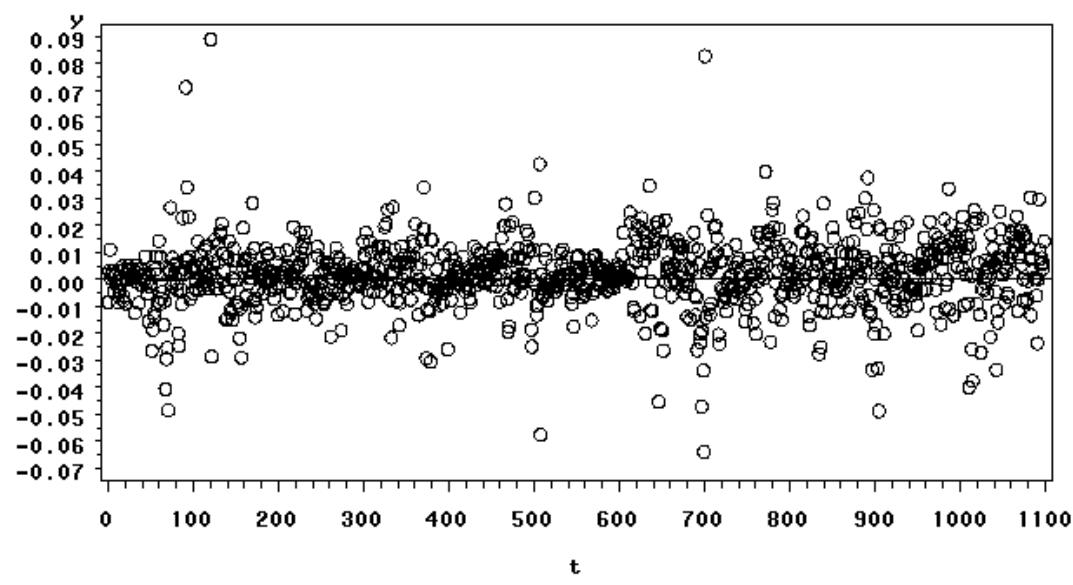

Figura 2: Rentabilidad IGBC.

Obteniendo los siguientes resultados:

Primera iteración

$\beta_{0}=0.0016218$

$\beta_{1}=0.2075$

Segunda iteración

$\beta_{0}=0.0012744$

$\beta_{1}=0.3738$

tercera iteración

$\beta_{0}=0.001274$

$\beta_{1}=0.4904$

Cuarta iteración

$\beta_{0}=0.00046085$

$\beta_{1}=0.7722$

Quinta iteración

$\beta_{0}=0.001037$

$\beta_{1}=0.5198$

Sexta iteración

$\beta_{0}=0.001034$

$\beta_{1}=0.4851$ 
Se observa que tanto en la quinta como en la sexta iteración, los valores de los componentes de la matriz de estados convergen a valores similares, lo cual es una indicación de que estos son los valores óptimos para el filtro. El comportamiento de la serie (filtrada) permite analizar la estructura original del proceso eliminando ruido y observaciones que pueden contaminar las estimaciones del proceso. Mediante la siguiente grafica se observa el comportamiento general de la serie.

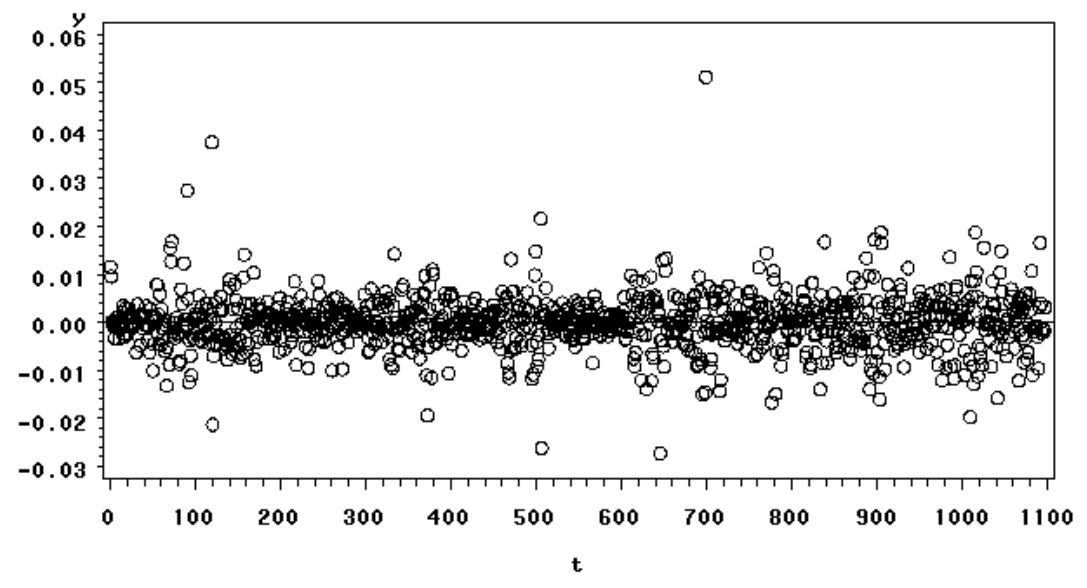

Figura 3: Filtro Kalman para IGBC.

Estos valores pueden ser reemplazados en la matriz de estados $G$ y con ellos a partir del conjunto de información $I_{t}$, es posible encontrar valores para los tres casos abordados para el filtro de Kalman. El primero en donde la solución al problema de predicción ofrecerá estimaciones que son extrapolaciones hacia el futuro de los componentes del vector de estado; por otra parte, el filtrado permitirá actualizar las estimaciones del estado no observable a medida que se dispone de una nueva observación y finalmente, el suavizado ofrece la mejor estimación del estado en cualquier instante del tiempo con toda la información de la muestra.

\section{Conclusiones}

La metodología Box-Jenkins para analizar series de tiempo con problemas de nivel y el analísis de variables latentes, presenta varias limitantes tales como eliminar observaciones en el proceso de diferenciación. Sin embargo, la metodología que emplea el modelo de estados resuelve algunos de estos inconvenientes.

La representación en el modelo de estados de la serie, permite analizar el comportamiento de variables latentes y a su vez el Filtro Kalman permite analizar procesos en los cuales la función de máximo-verosimilitud no es conocida, además permite encontrar estimaciones para procesos que no poseen normalidad en los residuales. 
El ajuste de modelos autoregresivos en forma iterativa, permite adaptar técnicas clásicas del análisis de series de tiempo con procedimientos iterativos.

\section{Agradecimientos}

Agradecemos a los evaluadores por los valiosos comentarios hechos al documento.

Recibido: 8 de diciembre de 2009

Aceptado: 17 de marzo de 2010

\section{Referencias}

Aydemir, A. (1998), 'Forecast performance of threshold autoregressive models - a monte carlo study', UWO. Department of Economics Working Papers .

Black, F. \& Scholes, M. (1973), 'The pricing of option and corporate liabilities', Journal of Political Economy .

Bollerslev, T. (1986), 'Generalized autoregressive conditional heteroskedasticity', Journal of Econometrics .

Breidt, F., Crato, N. \& de Lima, P. (1998), 'The detection and estimation of long memory in stochastic volatility', Journal of Econometrics .

Campbell, J. \& Hentschel, L. (1992), 'An asimetric model of changing volatility in stocks returns', Journal of Financial Economics .

Davis, M. \& Vinter (1985), Stochastic Modelling and Control, Chapman and Hall.

Durbin, J. \& Harvey, A. (1985), The effects of seat belt legislation on road causalties in great britain: Report on assessment of the statistical evidence, Technical report, Complusory seat belt wearing report by the Department of Transport.

Engle, R. (1982), 'Autorregressive conditional heteroskedasticity with estimates of united kingdom inflation', Econometrica .

Fama, E. (1965), 'The behaviour of stocks market prices', Journal of Business .

Figlewski, S. (1997), 'Forecasting volatility', Financial Markets, Institutions and Instruments

Garman, M. B. \& Klass, M. (1980), 'On the estimation of security price volatilities from historical data', Journal of Business .

Geert, B. \& Campbell, H. (1994), 'Time-varing world market integration', NEBER Working Papers (4843). 
Hannan, E. \& Deistler, M. (1988), La Teoría Estadística de Sistemas Lineales, Wiley.

Harvey, A. (1994), Forecasting structural time series models and the kalman filter, Cambridge University Press.

Hsieh, D. (1995), 'Nonlinear dinamics in financial markets: Evidence and implications', Financial Analysts Journal .

Li, C. \& Li, W. (1996), 'On a double threshold autoregessive conditional heterokedasticity time series model', Journal Applied Econometrics .

Mandelbrot, B. (1963), 'The variation of certain speculative price', Journal of Business .

Markowitz, H. (1952), 'Portafolio selection', The Journal of Finance .

Merton, R. (1974), 'On the pricing of corporate debt: The risk structure of interest rates', Journal of Finance .

Rabemananjara, R. \& Zakoian, J. M. (1993), 'Threshold arch models and asymmetries in volatility', Journal of Applied Econometrics .

Sharpe, W. (1964), 'Capital asset prices - a theory of market equilibrium under conditions of risk', Journal Of Finance .

Shephard, N. (1986), Time Series Models in Econometrics, Chapman and Hall.

Steyn, I. (1996), 'State space models in econometrics: a field guide', Vrije Universiteit.

Taylor, S. (1986), Modelling Financial time series, John Wiley.

Tobin, J. (1958), 'Liquidity preference as behavior towards riks', Review of Economic Studies .

Tong, H. (1978), On a Threshold Model.

\section{A. Implementación del procedimiento en SAS}

Para calcular las frecuencias teóricas

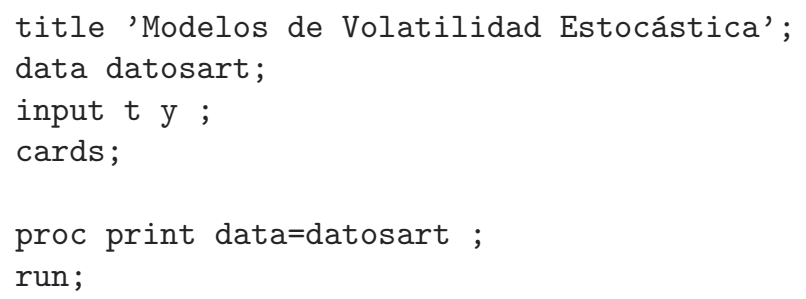




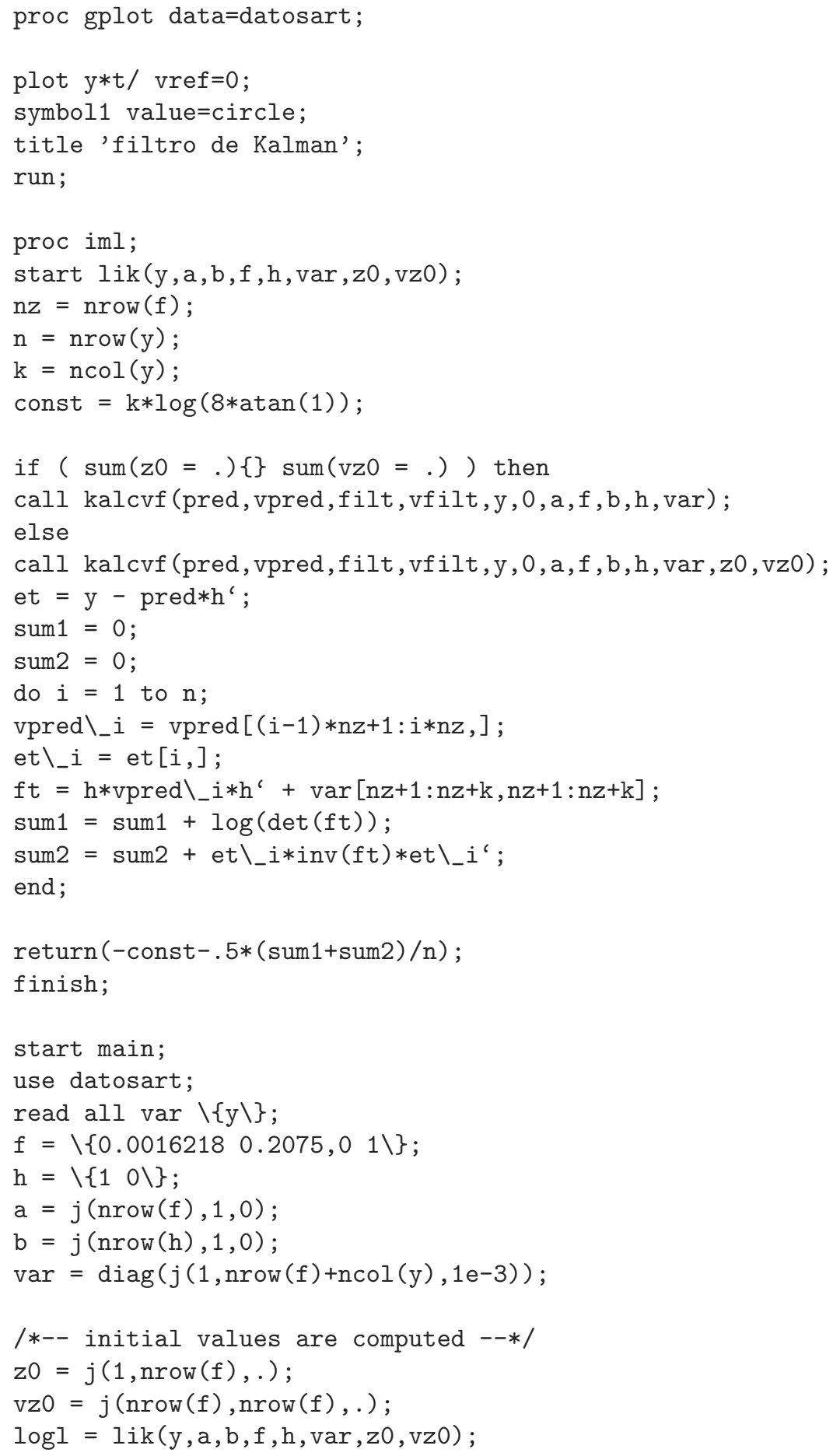




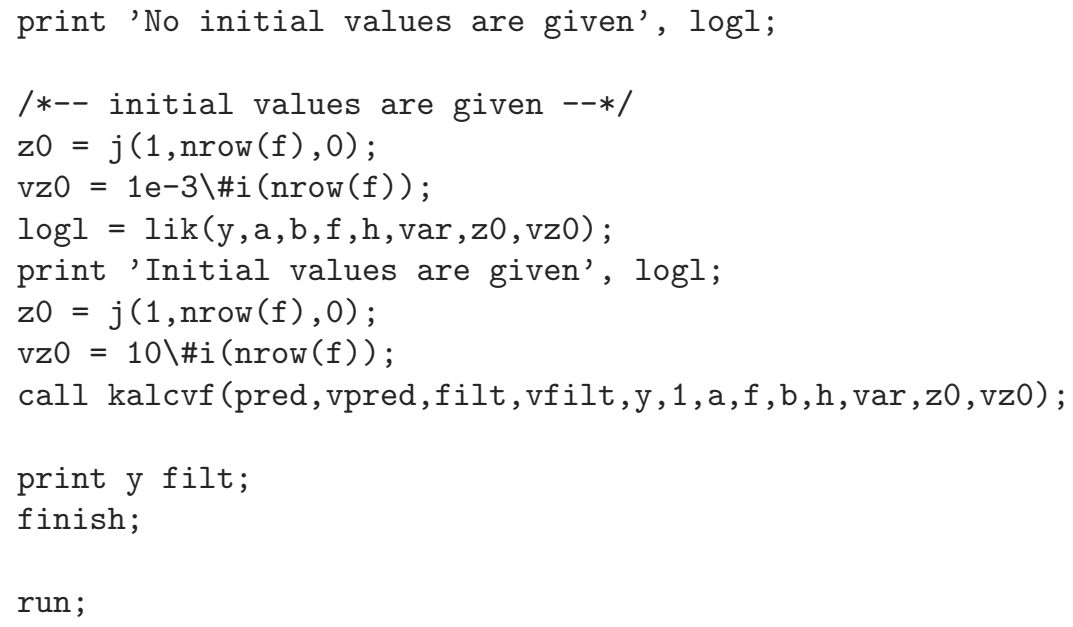

